\title{
DESIGN AND DEVELOPMENT OF AN INTELLIGENT DYNAMIC PATH FINDING/SURVIVALLENCE AUTOMATED GUIDED VEHICLE USING NI MYRIO
}

\author{
Bharathi. $\mathbf{G}^{1}$, Phanindra Reddy ${ }^{2}$ \\ ${ }^{1}$ M.Tech IV SEM, Dept of ECE, RYMEC Bellary \\ ${ }^{2}$ Associate Professor, Dept of ECE, RYMEC Bellary
}

\begin{abstract}
Automated Guided Vehicles (AGV) are emerging technology in many fields. These vehicles are used as advanced technology to help in the process of automations such as wild animal monitoring, military, mobile transportation and underwater exploration. There are various methods of path planning which are Global and Local Path Finding. In this paper GPS connectivity mapping is done with the help of myRIO device and android mobile. Collision avoidance is done with the help of Ultrasonic sensor which is integrated with LabVIEW for controlling and feedback operation. The objective of this paper is to design a prototype using myRIO device and LabVIEW software that will provide exact location and condition of AGV. Here we are developing an inexpensive, mobile and intelligent AGV prototype that will be interfaced with LabVIEW and with the help of a device called as NI myRIO.
\end{abstract}

Keywords: NI myRIO, AGV, LabVIEW, Path Finding

\section{INTRODUCTION}

Automated Guided Vehicles (AGV) has been applied for the flexible manufacturing system. Many researchers developed and designed it in order to suite with their applications which are related to the main problem of factory. AGV works on a predefined guideline. Steering control of mobile robot is done using GPS based on mobile location. The various ways for defining the path include optical, magnetic, laser guided, GPS control etc. Our objective here is to develop a prototype of an AGV using myRIO device and virtual instrument environment software LabVIEW.

Robotics is known as a new revolution to the entity of beings that varies according to its uses. In modern day environments, robotics and automation are implemented in wild animal monitoring, military, medical practice, construction, outer-space exploration, household assistance, mobile transportation and quite recently, underwater exploration and also in detecting the forest fire.

Most of intelligent manufacturing systems have several ways in order to enhance the overall performance of production rate. One way in manufacturing system was adopted the automatic guide vehicle (AGV) into more flexible for obtaining high throughput. Automated Guided Vehicles (AGV) has been applied for the flexible manufacturing system. Many factories were adopted it into assembly line or production line such as automobile, food processing, wood working, and other factories. Many researchers developed and designed in order to suite with their applications which are related to the main problem of factory. Automatic Guided Vehicle (AGV) has firstly developed and conducted the research by [17-18-19] in the attempt to using at Jumbo Truck
Monitoring wild animals, especially those that are becoming endangered (for example, lynxes and wolves) is important for biology researchers. Solutions for the monitoring already exist; however, they all have drawbacks, such as limited range or lifetime, sensing modality, re-porting delays, unreliability of operation. In this work we describe our experiences in designing an improved animal monitoring sensor system and low-level software for sensor node control and communication. The target animals for this particular research are wild lynxes or canines, however it can be extended to other animal species.

Currently there has been study of automated guided robots which is used in wild animal monitoring to understand the reasons behind some species of the wild animals getting endangered. Automated Guided Vehicle(AGV) Intelligent Dynamic Path Planning is one of the important areas of interest in robot's offline decision making to continuous monitoring of wild animal activity.

In this proposal an automated guided vehicle which is a driverless mobile robot uses a path planning approach for remote AGVs. The objective is to develop a prototype of an AGV using NI myRIO embedded controller and virtual instrument environment NI LabVIEW real time software and also describes development of a graphical driver interface (GDI), based on the web server function of the NI myRIO, implemented via Wi-Fi and myRIO host connection with a computer.

\section{AUTOMATED GUIDED VEHICLE}

$\mathrm{AGV}$ is one type of Material Handling Equipment (MHE) like conveyors, cranes \& hoists, elevator \& lifts, automatic storage \& retrieval system and so on which are focuses on 
process of transferring something from one place to another places especially in industrial sector or industrial warehouse. Actually, the goals to maintain or improve product quality reduce damage and provide protection of materials, promote safety and improve working condition, promote productivity, control inventory and so on. For further information, AGV is a driverless vehicle capable of moving along predetermined paths and performing certain prescribed duties. AGVs have become increasingly popular as a means of horizontal material handling transportation system. They are used wherever there is a need for an autonomous transportation system. AGVs are particularly useful where products need to be handled carefully or the environment is potentially dangerous to humans. Examples include handling of telecommunication products, IC chips, voltage cables and radioactive materials. In the automotive manufacturing industry, AGVs have been combined with robots to perform welding and painting operation. Sensors identified for the construction of the $\mathrm{AGV}$ in this project are ultrasonic sensors, temperature, pressure etc.

\section{Components of an AGV System:}

$\checkmark$ Vehicles: The vehicle or the AGV consists of the frame, batteries, on-board charging unit, and electrical system, drive unit, steering, precision stop unit, communication unit, safety system and work platform. The components mentioned above can each be further classified into different categories based on their capabilities and features.

$\checkmark$ Guide path and guidance systems: The guide path techniques used are known as passive or active tracking. Passive tracking occurs when optical or metal detection principles (wireless) are used for vehicle guidance whereas active tracking involves inductive principles (for example, guide wire is used to help tracking).

$\square$ Floor and system controls: The controller is the brain of the whole system, tying the vehicle and the guide path together and integrating the system. The AGVs contains three levels of control architecture: vehicle control system, floor control unit and vehicle on-board processor.

\section{METHODOLOGY}

The AGV also finds useful in an environment where obstacle detection/avoidance is very much essential, also the AGV's navigation is quiet flexible using either the predetermined path or by using maps dynamically, and dual power mode (battery/photovoltaic) system allows the AGV to work on solar and battery power. The sensors used in AGV gather the surrounding atmospheric parameters like temperature, pressure, $\mathrm{Co} 2$, etc. The camera embedded in the AGV helps to indentify natural landmarks and nearby obstacles. The block diagram of this proposal is as shown in the figure 1 .

Initially the overall data is collected by various sensors and given to the myRIO. Further the same data is sent to the LabVIEW where the required operations are done about the location finding. The map gives the various location points to the myRIO device and the solar panel is used to give the light at night times. The motor driver units are helped for the robot movement.

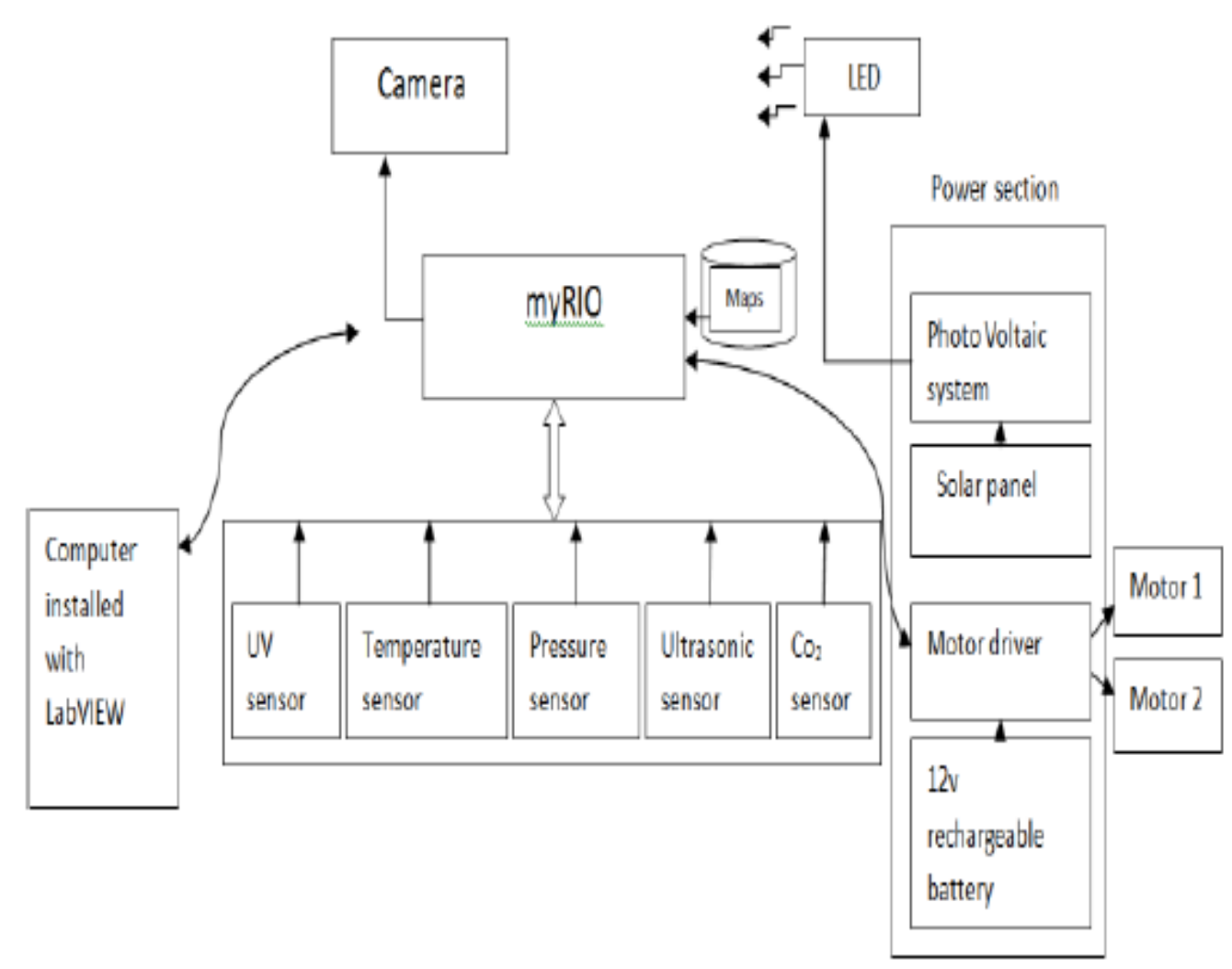

Fig 1: Block Diagram of Dynamic Path Planning using NI myRIO 


\section{Possible Outcomes}

1. Continuous monitoring of endangered wild animal activity provides the crucial reasons behind the animal getting endangered.

2. By using sensors monitoring of animals habitat provides concrete statistics on the environment in which it lives in.

3. Navigation of AGV is done dynamically using Google maps/GPS. This is very much useful in following the animal and also to manoeuvre the AGV.

4. In a different context, example in military using Gas sensors and camera it is possible to provide the firsthand information about the enemy whereabouts in a hostile building/environment.

5. Gas sensors helps in sensing various poisons gases that may kill/suffocate the soldiers during retaliation.

6. Camera provides either images or continuous video of the hostile environment which is handier in knowing the enemies exact location during their hideouts.

\section{CONCERNED WORK}

There is an increasing demand to understand the reasons behind some species of the wild animals getting endangered; the reasons can be better understood by continuously monitoring the endangered animal's daily activities and its environmental parameters. This proposed Remote AGV using NI myRIO possess the characteristic to be dynamically steered using Google maps \& GPS, also embedded with camera and other atmospheric parameters measurement sensors not only helps in monitoring the animals and the environment continuously but also provides enough data to understand the potential threats to the species.

There exists an extensive need for remote AGV in military area, for instance to gather the first hand information about the hostile environment/building, where it is potentially dangerous to soldiers. The camera on the AGV provides the soldiers with the structure of the building (viz. Building Entry/Exits), the position of the enemies inside the building using the dynamic navigation facility, also using the available sensors the AGV senses the indoor conditions for any hazardous gases, temperature etc,.

\section{OBJECTIVES}

The objective is to develop a prototype of an AGV using NI myRIO embedded controller and virtual instrument environment NI LabVIEW real time software, And also to find out the reasons behind some species of the wild animals getting endangered; the reasons can be better understood by continuously monitoring the endangered animal's daily activities and its environmental parameters.

\section{ADVANTAGES OF USING LabVIEW}

- The code can be compiled to generate distributable EXEs.

- $\quad$ Flexible, powerful and scalable design (can open and connect to third party tools along with external libraries).
- Easy to Learn, maintain, use and upgrade (using graphical constructs and intuitive graphical programming).

- Use of one tool for design, prototyping and deployment.

- Can be used in Multidisciplinary fields (easy VI programming language for different applications and expert use in different disciplines of science and engineering).

- Good hardwaresoftware integration (supports wide range of data acquisition and embedded devices).

- Multiplatform (Mac OS, Linux, Windows, RTOSs etc.)

- Easy communication with instruments using serial, Bluetooth or wireless communication.

- Real time solving and execution of complex algorithms.

- Acts as the Bridge to industry - nowadays many industries are using LabVIEW as the control system software for controlling various processes in the plant.

- Help to develop better and faster algorithms.

- $\quad$-Shorter time to prototype, discovery and deployment to market.

\section{EXPECTED RESULTS}

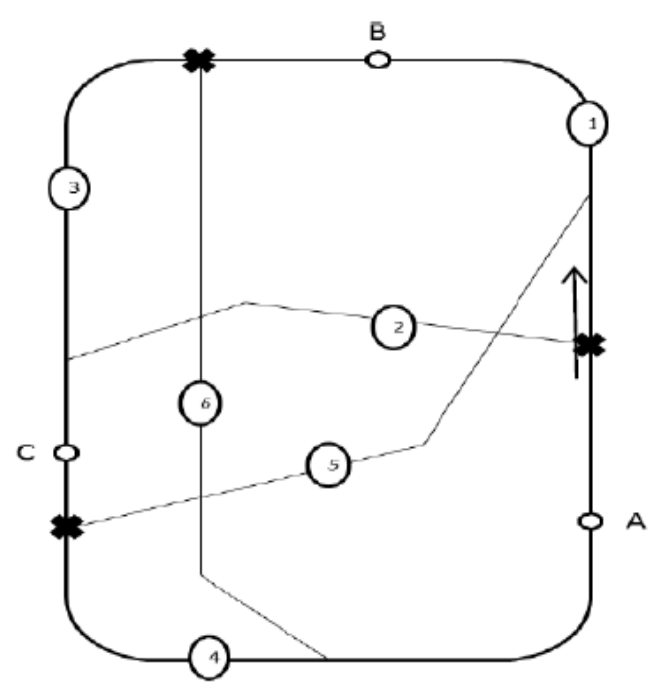

Fig 2: Map of a guided path

The guided path map consists of three stations (A, B and C). There are six paths numbered from 1-6 which formed by interconnecting the three stations. There are crossovers at certain points (indicated by $\mathbf{X}$ )in the path where the AGV will turn by approximately 90 degree to go from one station to other station skipping the middle station in between these two stations. E.g. In case we want to go from station A to station $\mathrm{C}$ directly bypassing the station $\mathrm{B}$, the $\mathrm{AGV}$ commands will be like this, First the AGV will start from station A following the path 1, in the middle as it wanted to go to station B it will deviate from path 1 to path 3 to go to station $\mathrm{C}$ through intermediate path 2 . For finding out which path it should take, a crossover , $\mathrm{X}^{\mathrm{ee}}$ is used as the reference point on the map. Thus the AGV will achieve motion from Station A to Station C skipping the Station B between. 
For seeing the results we observed when the AGV was going from station $\mathrm{C}$ to station $\mathrm{A}$, we changed the destination station to B by modifying the value in LabVIEW VI. The AGV which was following the path 4 now got to know that it has to go to path 1 to reach the station $B$; it started to scan for a crossover point. When the AGV got the location of the crossover point it turned approximately by 90 degrees and moved to path 1 through traversing the path 5 . Thus the other stations can be traversed by using the path selection table as shown in Figure 2.

\section{CONCLUSION}

Automated guided vehicles' application in production facilities increases continuously. As these vehicles count as the industrialized version of mobile robots, the later ones can be regarded as the future of industrial AGVs. Landbased mobile robots currently use sensors for general navigational purposes, identification and range finding. The aim of this research work was to show a possible development area for AGVs' sensor, which is intelligent but cost-effective as well. Further the developed method shows new possibilities for visual serving of these industry material transport vehicles.

Thus a cost effective means of employing powerful programming tool for various applications using prototyping myRIO is developed. Potential of this method will be extended for various other engineering applications in near future.

\section{REFERENCES}

[1]. Anjay Prasad, Vishal Prakash Halale, Siddhant Vinod Bora," A Model Based Approach for Algorithm Development in Real Time Operation of Automated Guided Vehicle Using Labview" (C) 2014 IJEDR | Volume 2, Issue 3 | ISSN: 2321-9939.

[2]. Mohd Azlan Shah Abd Rahim, Illani Mohd Nawi, "Path Planning Automated Guided Robot", Proceedings of the World Congress on Engineering and Computer Science 2008, San Francisco, USA, WCECS 2008, October 22 - 24, 2008.

[3]. Reinholds Zviedris1, Atis Elsts12, Girts Strazdins12,Artis Mednis12, and Leo Selavo12."Wild Animal Monitoring Using Sensor Networks".

[4]. NurulHuda Binti Muhamad Nasir "Mechanical Design of an Automated Guided Vehicle (AGV)" June 2006.

[5]. Fahad Ullah, Qaiser Habib, Muhammed Irfan, and Khawaja M. Yahya "Autonomous Vehicle Guidance and Control using OpenStreetMap and Advanced IntegrationTechniques" International Journal of Computer Theory and Engineering, Vol. 3, No. 5, October 2011.

[6]. Ralf w.seifert, Michael G.Kay,James R.Wilson "Evaluation of AGV Routing Strategies Using Hierarchical Simulation" proceedings of the 1995 winter simulation conference ed.calxopoulos, K.Kang ,W.R.Lilegdon, and D.Goldsman.

[7]. Sajjad Yaghoubi, Sanam Khalili3, Reza Mohammad Nezhad4, Mohammad Reza Kazemi5 \&Mahsa Sakhaiifar5 "Designing and Methodology of Automated Guided Vehicle
Robots/ Self Guided Vehicles Systems, Future Trends" IJRRAS13(1) October

[8]. Reinholds Zviedris1, Atis Elsts12, Girts Strazdins12,Artis Mednis12, and Leo Selavo12. "Wild Animal Monitoring Using Sensor Networks"

[9]. G. Reccari Y. Caselli F. Zanichelli A. Calafiore "Vision-based Line Tracking and Navigation in Structured Environments" Dipartirriento di Ingegneria dell'Informazione IJniversity of Parma. - Italy. 